spindle formation, has been shown to allow the preparation of the karyotype of the penetrating spermatozoon (variably more than one per egg), of a clarity sufficient for evaluation of the numbers and the character of the sperm chromosomes. With wider establishment of this technique, studies of the possible incidence with which spermatozoa act as primary agents of the chromosome imbalance so common in early human embryopathies, and the degree to which environmental hazards can affect sperm chromosomes, would seem to be on the horizon. Whatever the conditions of culture, however, the 'humster' zygote fails after one cell division at the two-cell stage. Even in a world where bacteria are coerced to produce human insulin, small boys with furry brown ears are not a foreseeable prospect!

\title{
Understanding natural killer cells
}

\section{from Peter Beverley}

NATURAL KILLER (NK) cells were first identified by their ability to kill, without deliberate prior immunization, certain tumour target cells grown in vitro. Otherwise they were defined only in negative terms: that is, they were not thymus-derived (T) or bone marrowderived (B) lymphocytes, nor were they adherent or phagocytic. Though their origins were thus rather mysterious, they were at first widely presumed to be the mediators of immunosurveillance against tumours $^{1}$. Subsequent studies have suggested however that NK cells may be related to either the $\mathrm{T}^{2}$ or myeloid ${ }^{3}$ lineages and may have an immunoregulatory role ${ }^{4}$. Furthermore, several authors have demonstrated heterogeneity of NK effector cells, and that different target cells are differentially susceptible to these effector subpopulations ${ }^{5}$. In the face of this apparent confusion can it now be said that there is such an entity as an NK cell and is it possible to define its biological role?

Examination of mutant animals deficient in various cell types, such as the nude or beige mouse ${ }^{2}$, or of humans with genetically determined diseases ${ }^{6}$, has not settled the controversy over the origin and phenotype of NK cells. On the other hand, NK cells do have specific properties not characteristic of other lymphocytes. In the mouse NK cells have been identified by a serological marker ${ }^{7}$ and in man, although no unique surface marker has yet been defined, the more old fashioned, but not to be despised, use of cytological examination has revealed that most NK cells are large, granular lymphocytes with characteristic cytochemistry ${ }^{8}$. This is not to say that all cytotoxicity detected using cultured tumour lines in vitro as targets is due to this cell type. Certainly, activated lymphocytes which carry undoubted T-cell markers will lyse a variety of cultured target cells.

Peter Beverley is ICRF Senior Scientist at the ICRF Human Tumour Immunology Group, University College Hospital Medical School, London.
If many different cell types can lyse cultured tumour targets in vitro and if freshly isolated cells are in general insensitive to NK activity, what can be made of claims that NK cells operate in vivo as a surveillance mechanism against tumours? Several authors have shown that NK cells can mediate resistance in vivo against transplantable tumours. Many of these experiments are, however, difficult to interpret because NK cells cannot be purified. Kasai et al. (this issue of Nature p.334) have now adopted a rather different approach: they have used systemically administered antibody to asialo GM1 surface membrane glycolipid to block NK activity and shown a dramatic reduction in resistance to syngeneic tumour. The relevance of their results to natural tumour immunity is not entirely clear because they used a well established transplantable tumour cell line. The difficulty with such cell lines is that they generally contain tumour viruses and it may be against the viruses that the immune response is directed.

Another line of evidence that could be interpreted as indicating a role for $\mathrm{NK}$ cells in protection against virus infection is the observation that NK activity is increased by interferons ${ }^{9}$. Furthermore, antibody to interferon has been shown to block NK function in nude mice, and to allow the growth of xenogeneic cell lines even when these were persistently infected with viruse ${ }^{10}$. Such data are highly reminiscent of much older findings in mice immunosuppressed for long periods with antilymphocyte serum in which the tumours which arose were shown to be due to polyoma virus ${ }^{11}$. Taken together, these results may be more consistent with a view of the NK cells as a surveillance mechanism predominantly directed at viruses not tumours. This line of reasoning suggests that therapeutic manoeuvres, such as administration of interferon, which may activate NK cells, will be most effective against virus-induced tumours. In man, the successful use of interferon to treat laryngeal papilloma may be an example of this $^{12}$.

The increase in NK activity mediated by interferon results at least partly from recruitment of previously inactive cells. But interferon also affects target cells: they become more resistant to lysis and have been shown to express more major histocompatibility complex (MHC) antigen. This latter effect, while it may not account for the change in susceptibility to NK cells, might well make virus-infected cells more susceptible to $\mathrm{T}$ cell-mediated killing because of the importance of $\mathrm{MHC}$ antigens as target structures for T cells.

This raises the question of whether the function of NK cells need necessarily be the killing of tumour cells. Other data suggest a regulatory role: interferon may be produced by NK cells themselves, and play a part in the generation of suppressor $T$ cells $^{13}$. This implies that NK cells are an integral part of the immunological network of $T$ and non-T cells. Since immunologically active cells are regulated by multiple signals (MHC antigens, specific antigens, helper and suppressor factors), it is not surprising that NK regulation has now been shown to depend on signals other than interferon: Henney et al. (this issue of Nature, p.335) have shown that their activity is augmented by interleukin-2. Whether this is a direct effect or mediated indirectly via another cell type is not established. It thus remains possible that the final mediators of the augritentation are interferons produced by intermediary cells induced by interleukin-2. This would imply synergistic effects of different interferons on NK cells. Irrespective of the details of the mechanism of the interleukin-2 effect however, this finding once again places the NK cell firmly in the immunological network.

In summary, recent data seem to be leading away from earlier and rather sterile arguments about the phenotype and effector specificity of NK cells (although these remain important unresolved questions) and towards a deeper understanding of the biology of NK cells in vivo and in vitro. The new tools available, monoclonal antisera ${ }^{14}$, in vitro grown normal ${ }^{15}$ and tumour ${ }^{16}$ cell lines and purified mediators, should allow rapid progress.

\footnotetext{
1. Roder, J.C. \& Haliotis, T. Immun. Today 1, 96 (1980).

2. Herberman. R.B. el al. J. Immun. 121, 304 (1978)

Roder, J.C. \& Duwe, A Nature 278, 451 (1979).

4. Hansson, M., Kiessling, R. \& Andersson, B. Eur. J. Immun. 11, 8 (1981).

5. Knight, R.A. \& Fitzharris, P.F. Br. J. Cancer 42, 243 (1981).

. Roder, J.C. et al. Nature 284, $553(1980)$

7. Glimcher, L., Shen, F.W. \& Cantor, H. J. exp. Med. 145, 1 (1977).

8. Saksela, E. et al. Immun. Rev. 44, 71 (1979).

9. Trinchieri, G. \& Santoli, D. J. exp. Med. 147, 1314 (1978).

10. Reid, L.M. el al. Proc. nain. Acad. Sci. U.S.A. 78, 1171 (198i).

11. Simpson, E. \& Nehlsen, S.L. Clin. exp. Immun. 9. 79 (1971).

12. Dunnick, J. J. infect. Dis. 142, $293(1980)$

13. Kadish, A.S. et al. J. exp. Med. 151, 637 (1980).

14. Secker, D.S. \& Burke, D.C. Nature 285, $446(1980)$

15. Denneri, (i. Nature 287, 47 (1980).

16. Johrson, D.R. \& Jondal, M. Nature 291, 81 (1981).
} 